\title{
Experience with Tetanus in a Tertiary Hospital in South East Nigeria
}

\author{
*Onwuekwe, I 0 MBBS FWACP ${ }^{* *}$ Onyedum, C C MBBS MWACP ${ }^{* * *}$ Nwabueze, A C MD FWACP FMCP FRCP (Ed) \\ Department of Medicine, University of Nigeria, Teaching Hospital, Enugu, Nigeria
}

\begin{abstract}
Background: Tetanus has for long been an avoidable source of morbidity and mortality particularly in developing countries. The aim of this study was to review the experience with managing tetanus in a regional tertiary hospital in Nigeria.
\end{abstract}

Methods: A retrospective study of patients who were admitted with a clinical diagnosis of tetanus between January 1999 and December 2003 was done. Relevant data were extracted from the patients' case records.

Results: A total of 12 patients were identified within the time frame with a diagnosis of tetanus. There were more male than female patients (ratio 1.4:1) with city dwellers constituting a small majority. The mean age of the patients was 29.8 years. Lower limb injuries accounted for the portal of entry in $75 \%$ of cases while one case followed a practice of oral sex. All patients were managed in the general ward and no mortality was recorded despite the presence of dysautonomia in some patients.

Conclusion: Despite the high rate of morbidity and mortality associated with tetanus in developing countries and despite some regional differences in presentation, careful clinical management even in resource poor countries can result in verygood outcomes.

\section{Date accepted for publication: $10^{\text {th }}$ October 2007 Nig J Med 2008; 50 - 52 copyright@2008 Nigérian Journal of Medicine}

\section{INTRODUCTION}

Tetanus persists as a global health problem despite the efforts of the World Health Organization. Though a preventable disease, it is a neurological emergency with an estimated annual global incidence of 1 million cases and a mortality rate of $50 \%$.

In Nigeria, tetanus is a major cause of high mortality in both children and adults, ${ }^{2}$ and this is despite the vigorous immunization campaigns of the last one to two decades.

We conducted this retrospective review to be able to determine the pattern of presentation, case fatality rate and factors influencing mortality with a view to mapping control strategies.

\section{PATIENTS, MATERIALS AND METHODS}

The University of Nigeria Teaching Hospital Enugu is a 760-bed facility that serves as a tertiary level referral center for mainly the South East of Nigeria. It also receives patients from other parts of the country.

The South East Nigeria is principally a rain forest region with the population principally engaged in agriculture, public/civil service and commerce/ trading activities.

All adult patients (age $>16$ years) with a clinical diagnosis of tetanus admitted into the medical wards of the University of Nigeria Teaching Hospital from January 1999 to December 2003 were retrospectively retrieved and data was analyzed to determine the demographic features, clinical details, management and outcome of treatment.

\section{RESULTS}

A total of 12 patients were admitted during the period under review. All the patients were diagnosed in the emergency room by clinical examination.

There were 7 males and 5 females. Their ages ranged from 18 years to 59 years with a mean age of 29.83 years. The female patients had a mean age of 29.80 years with a range of 18 years- 46 years while the male patients had a mean age of 29.86 years with an age range of 19 years- 59 years. Table 1 shows the age distribution of the patients.

Seven of the patients were urban dwellers while five were villagers. Half of the patients $(n=6,50 \%)$ were engaged in trading and/or other commercial activities while three $(25 \%)$ were students ( 2 in tertiary institutions and 1 in secondary school). There were two farmers $(16.7 \%)$ involved while only one patient $(8.3 \%)$ was a civil servant.

All the patients had an identifiable source of the infection. The portal of entry involved the limbs in nine patients (six patients with lower limb injuries and three patients with upper limb trauma). Incidentally one of the patients with upper limb trauma had associated head 
injury. The remaining three patients consisted of single cases respectively of post-convulsive burn, penile laceration and oropharyngeal ulcers (consequent upon oral sex). Table 2 shows the distribution of the sites of infection in the patients.

\section{Table 1: Showing age distribution of patients}

\begin{tabular}{ll|c}
\hline Age Range (years) & Number \\
\hline 15 & 24 & 6 \\
25 & 34 & 2 \\
35 & 44 & 2 \\
45 & 55 & 1 \\
Above 55 & 1 \\
\hline Total & $\mathbf{1 2}$ \\
\hline
\end{tabular}

The incubation period ranged from 11 days to 98 days while onset time ranged from 24 hours to 6 days. All the patients $(n=12)$ presented with generalized tetanus (with trismus, trunk and limb spasms). Majority of the patients ( $\mathrm{n}$ $=10,83.3 \%$ )had no autonomic involvement (dysautonomia) while only two patients featured alterations in heart rate, paroxysmal hypertension and diaphoresis.

All the patients were managed in the general medical ward. None was transferred to the intensive care unit (ICU) of the hospital. To control spasms, pharmacologic intervention consisting of a combination of phenobarbitone, chlorpromazine and diazepam was used in 9 patients $(75 \%)$ while 2 patients $(16.7 \%)$ were managed with a combination of chlorpromazine and diazepam. Only one patient was managed solely with diazepam. The medications in all cases were administered parenterally.

All the patients received tetanus immunoglobulin in addition to the care of other associated clinical findings.

No mortality was recorded as all the patients $(n=12)$ made successful recovery and were discharged home. All the patients received the initial dose of tetanus toxoid as active immunization on discharge. No record was obtained as to subsequent doses.

Table Il: showing the distribution of portals of infection

\begin{tabular}{l|c}
\hline \multicolumn{1}{c|}{ Wound } & Number of patient \\
\hline Limb injury & 9 \\
Post burn & 1 \\
Penile laceration & 1 \\
Oropharyngeal ulcers & 1 \\
\hline Total & $\mathbf{1 2}$ \\
\hline
\end{tabular}

\section{DISCUSSION}

The total number of 12 cases of tetanus seen in our 5 year review is low when compared with other review studies conducted in centres within and outside Nigeria. ${ }^{3,-7}$ However we think that improved immunization strategies amongst the populace coupled with the urban setting of the hospital may account for the rather low figure encountered.

The male preponderance in our study agrees with the results of previous studies in Nigeria. ${ }^{3,5}$ The greater involvement of men in activities with a high risk of bacterially contaminated injuries for example agriculture and mechanical work may be responsible for this pattern.

In studies in South West Nigeria and Ghana, the youths were predominantly affected by tetanus. Arogundade and Bello et al as well as Oladiran and Mieir et al working in Ile Ife and Ogbomosho (all in South West Nigeria) recorded mean ages, for tetanus patients, of 35.96 years and 26 years respectively. ${ }^{3,5}$ Hesse's group in Accra documented a peak age range of 20-29 years in affected patients. ${ }^{6}$ The mean age in our study was 29.83 years and this is consistent.

Greater attention to this age group especially in terms of health education and knowledge of preventive strategies is recommended. It is note worthy that in some studies out side of Africa, tetanus was found to affect mostly older people. Persons aged 60-65 years had the highest number of tetanus cases in Malaysia, while in Turkey a mean age of 46 years was recorded for tetanus patients. ${ }^{8,9}$ This pattern was also obtained in Brazil and Australia ${ }^{7,8}$ and may be as a result of poorer tetanus immunization status for people in this older age group.

Once again it is plausible to ascribe the predominance of urban dwellers over rural dwellers in this study to the city setting of the hospital. This finding contrasts sharply with the picture obtained in other reviews ${ }^{5,7,9}$ where tetanus patients were most often resident in the rural / village setting.

Though it is recognized that in as many as $20 \%$ of tetanus patients, it may not be possible to identify a possible source of infection or portal of entry, all the patients in this series had identifiable sources of infection. Lower limb injuries were the commonest sites of infection and this was consistent with the findings by other workers in South West Nigeria. ${ }^{4,5}$ 
Dysautonomia is a well-recognized risk factor for mortality from tetanus. ${ }^{5,9,11}$ In the lle lfe study, ${ }^{5}$ dysautonomia was a documented finding. However other studies from Benin ${ }^{4}$ and Ogbomosho ${ }^{3}$ in the mid and south western parts of Nigeria respectively did not report any such feature. In our series dysautonomia was evident in a minority of the patients $(16.7 \%)$. The relative rarity of dysautonomia may be a widespread finding in Nigerians and more studies are needed for further elucidation of this phenomenon.

The incubation periods and times to onset of spasms in our study are generally in keeping with standard estimates.

Whilst all the patients reviewed had generalized tetanus and were managed in the open general ward, it is worthy of note that no mortality was recorded. Though it is plausible that this significant outcome was the result of good clinical management including the use of optimal doses of parenterally administered muscle relaxants, we are constrained to note that tetanus cases in other centers in Nigeria often have a considerable degree of mortality. Mortality rates of $44 \%, 53.5 \%$ and 26.25 were recorded at Ogbomosho, ${ }^{3}$ lle-lfe ${ }^{5}$ and Benin ${ }^{4}$ respectively.

\section{REFERENCES}

1. Osuntokun BO. Perspectives in tetanus: the past two decades. A symposium on "Recent Advances in Neuro science". Dokita 1990; 19(1): 74-78.

2. Adeuja AOG, Osuntokun BO. Tetanus in the adult Nigerian: a review of 503 patients. EastAfr Med J. 1971; 48:653-691.

3. Oladiran I, Mieir DE, Ojelade AA et al. Tetanus: continuing problem in the developing world. World J Surg.2002 Oct; 26 (10): $1282-5$

4. Ogunrin OA, Unuigbe El. Tetanus: an analysis of the prognosticating factors of cases admitted into the medical wards of a tertiary hospital in a developing African country between 1990 and 2000. Niger Postgrad Med J.2004 Jun; 11(2): 97-102.

5. Arogundade FA, Bello IS, Kuteyi FA et al. Pattern of presentation and mortality in tetanus: a 10-year retrospective review. Niger Postgrad Med J.2004 Sep; 11 93): 198-202.

6. Hesse IF, Mensah A, Asante DK et al. Characteristics of adult tetanus in Accra. WestAfr J Med. 2003 Dec; 22(4): 291-4.
The relationship of these mortality figures to management in the Intensive Care Unit was not examined in these studies. Management of tetanus patients in the Intensive Care Unit has been shown to reduce the mortality rates in several countries . $^{7,8,10,12 .}$ However a different result was obtained in Honduras where despite intensive unit care, a mortality rate as high as $69 \%$ was recorded for tetanus patients and this was attributed primarily to the presence of dysautonomia. ${ }^{11}$

\section{CONCLUSION}

Tetanus remains a significant cause of death worldwide and this situation may not have been significantly altered despite widespread immunization efforts and the development of high- tech centers and Intensive Care Units.

Nevertheless a more aggressive approach to adequate immunization schedule should be encouraged. Closer attention to the provision of appropriate and effective clinical care of these patients even in resource poor countries may go a long way in reducing the unacceptably high mortality rates.

7. Litvoc Julie, Leite RM, Katz G. Epidermiology of tetanus in Sao Paulo State (Brazil). Rev Inst Med Trop Sao Paulo. 1991 Nov Dec; $33(6)$ 477-84.

8. Lau LG, Kong KO, chew PH. A 10-year retrospective study of tetanus at a general hospital in Malaysia. Singapore Med J.2001 Aug; 42(8): 346-50.

9. Saltoglu N, Tasova Y, Midikli D et al. Prognostic factors affecting deaths from adult tetanus. Clin Microbiol Infect.2004 Mar; $10(3):$ 229-33.

10. Newton-John HF. Tetanus in Victoria, 1957-1980.Review of 106 patients managed in one hospital. Med J Australia. 1984 Feb 18; 140(4): 194-200.

11. Orellanar San Martin, Su H, Bustamante Duvan D et al. Tetanus in an intensive care unit. Rev Neurol. 2003 Feb 15-28; $36(4): 327-30$.

12. Ebisawa I, Homma R. Tetanus in Japan; trends in mortality, case fatality and causes of death. Jpn J Exp Med. 1986 Aug; 56 (4): 155-61. 